\title{
Laser photobiomodulation: A new promising player for the multi-hallmark treatment of advanced cancer
}

\author{
Luis Santana-Blank ${ }^{1}$, Elizabeth Rodríguez-Santana ${ }^{1}$, Heberto Reyes ${ }^{1,2,3}$, \\ Jesús A. Santana- Rodríguez ${ }^{1}$, Karin E. Santana-Rodríguez ${ }^{1}$ \\ ${ }^{1}$ Fundalas, Foundation for Interdisciplinary Research and Development, Caracas-Venezuela \\ ${ }^{2}$ Hospital José María Vargas, Radiology Department, Caracas-Venezuela \\ ${ }^{3}$ Clínica Ávila, Radiology Department, Caracas-Venezuela
}

Received August 30, 2013; Revised September 2, 2013; Accepted September 3, 2013; Published Online September 4, 2013

\section{Scientific Note}

J. Watson, co-discoverer of the double helix structure of the DNA and a major force in cancer research over the last half century, has sharply criticized the War on Cancer on the following grounds: "Although the mortality from many cancers, particularly those of hematopoietic cells, has been steadily falling, the more important statistic may be that so many epithelial cancers (carcinomas) and effectively all mesenchymal cancers (sarcomas) remain largely incurable" 1 . Thus, Watson believes, that "the cancer world is not trying to cure incurable cancer. They need to concentrate on late-state disease $" 1-2$.

Indeed, efforts to increase awareness and screening have aimed to reduce late-stage disease and mortality. However, data show a significant increase in early-stages without a proportional decline in later ones ${ }^{3}$. Fortunately, most cancers do not progress to death. Still, cancer takes a heavy toll on society in terms of patient and family suffering, lost productivity and ever straining demands on public and personal finances. In this context, made worse by a "tsunami" of baby-boomers demanding more healthcare, ${ }^{4}$ laser photobiomodulation (L-PBM), alone or combined with standard agents, may prove effective at targeting advanced cancer and other complex diseases safely and, potentially, at a low cost.

A major obstacle for many cancer drugs aiming at single molecular targets to minimizing nonspecific toxicity has

Corresponding author: Luis Santana-Blank, MD;Fundalas Calle Las Flores con Guaraguao, C.C. Carabel PB Local 2, MUN 1262. Puerto La Cruz, Anzoátegui, Venezuela 6023-5; Email: luissantanablank@msn.com

Int J Cancer Ther Oncol, Vol. 1, No. 1, pp. 6-8, 2013.

This is an open access article distributed under the terms of the Creative Commons Attribution 3.0 License, which permits unrestricted use, distribution, and reproduction in any medium, provided the original work is properly cited. been that clinical response is often transitory and followed by relapse ${ }^{5}$. In light of this, and given that the acquisition of new-generation cancer hallmarks is intricately linked and made possible by the tumor microenvironment, it has been suggested that new anticancer therapies should not aim at single molecular targets to solely kill cancer cells, but at re-establishing homeostasis-homeokinesis, a micro-environment effect which, as will be discussed below, may be induced by light ${ }^{6}$.

L-PBM, also known as low-energy laser therapy (LLLT), refers to the use of monochromatic or quasi-monochromatic low-fluence light to induce primarily non-thermal photochemical effects. Skepticism surrounding this multidisciplinary field has been primordially rooted in a deep belief that, to affect biological systems, electromagnetic signals must ionize matter, or that too weak a signal may not be able to trigger biological effects ${ }^{7}$. Thus, a poor understanding of the physico-chemical basis of low-energy radiation and the fact that much initial research was methodologically flawed and came from behind the former Iron Wall deterred interest in the West for decades ${ }^{8}$.

More than three hundred worldwide publications in reputed peer reviewed journals have turned this scenario. It has been demonstrated that L-PBM can stimulate or inhibit cellular function ${ }^{9}$. It has also been ascertained that signal and target characteristics determine biological outcome, which is optimal (or even positive) only within a narrow set of parameters. Nonetheless, until recently, there was great trepidation to explore L-PBM in cancer due to fear of increasing tumor cell proliferation ${ }^{10-11}$.

Notwithstanding this, in cooperation with national and foreign research centers, our group completed animal testing and embarked on a phase I clinical trial in patients with advanced and progressive neoplasias using a singular low-energy infrared pulsed laser device (IPLD) that combines high-frequency ultrasound and near-infrared (NIR) radiation. Recruited patients, who suffered from advanced 
solid tumors, including epithelial cancers (carcinomas) and mesenchymal cancers (sarcomas), had exhausted available treatment alternatives and had a life expectancy $\geq 12$ weeks (TNM IV- UICC).

After > 10 years of follow-up, the IPLD was found to be clinically safe and to improve performance status and quality of life. Antitumor activity was found in $88.23 \%$ of patients ${ }^{12}$. Immune data from the same trial showed modulation of CD4 CD45RA+, CD25, TNF-alpha, and soluble IL-2 receptor (sIL-2R), ${ }^{13}$ in agreement with Coussens and others ${ }^{14}$. Also in accord with subsequent results by Tanaka et al., ${ }^{15}$ the cytomorphology results of the trial showed selective activation of programmed cellular death (i.e., apoptosis, necrosis, anoikis ) in neoplastic cells, but not in peripheral tissues. Microdensitometric T2-weighted MRI data further showed increased water content in tumor heterogeneities preceding tumor-volume reduction and therapeutic anticancer effects, ${ }^{16}$ showing that changes in water-content acted as early predicator of tumor response in a manner consistent with the approach of Ross, Chenevert and others ${ }^{17-18}$ for early tumor response determination.

Other studies show that L-PBM can trigger regenerative responses, alone or associated with stem cell therapy ${ }^{19}$. Epigenetic modulate chromatin structure, which affects gene transcription, ${ }^{20}$ and L-PBM has been shown to reduce the frequency of chromosome aberrations ${ }^{21}$. Clinical results further suggest that L-PBM can cause phenotypic changes 22 consistent with theoretical data from a nonlinear DNA model, in which chaotic behaviors generated by damping, external fields and torque in solitone dynamics induce open states of the DNA which can regulate transcription and replication $^{23}$. L-PBM has also shown effectiveness in the management of radiotherapy complications, such as mucositis ${ }^{24}$.

Structural, kinetic, and thermodynamic implications of the above findings have been documented by our group $25-27$. In addition, we have proposed detailed mechanisms that complement the work of numerous authors, ${ }^{28-30}$ and which help explain and substantiate one basic premise: that external electromagnetic energy (light) supplementation can enhance and even substitute for endogenous ATP production to power and modulate physiologically reparative mechanisms which can help reestablish homeostasis-homeokynesis, even when physiologic metabolic pathways have been compromised ${ }^{31}$.

Recently, based on studies by Pollack and others on the exclusion zone (EZ), described as a fourth phase of water, ${ }^{32}$ we hypothesized that the EZ might be targeted by L-PBM as an energy reservoir, which cells may use to fuel cellular work and trigger signaling pathways and gene expression in the presence of injury-induced redox potentials ${ }^{31}$. Nevertheless, we stressed that experimental proof that L-PBM would express effects via the EZ in a high-order biological system had not been attained. Now, clinical and experimen-

Copyright (c) Santana-Blank et al. tal results which are remarkably consistent with the current understanding of the EZ are in press ${ }^{33}$. Such evidence, and the growing substantiation and reproduction of the above results, lead us to be confident that L-PBM will have a bright future in medicine at large, and oncology in particular.

A major goal for L-PBM in cancer is to safely control programmed cellular death and differentiation, as suggested by referred studies and in accord with the need for new multiple-hallmark cancer therapies. Challenges include determining optimal treatment parameters and further documenting the underlying mechanisms for potential applications in oncology. However, and given that laser-based technologies can be significantly less expensive than most cancer drugs, we hope that L-PBM may soon help to lower treatment costs whilst raising standard of care and quality of life, particularly, for the most vulnerable, such as the elderly, the poor and those suffering currently-untreatable late stage disease.

\section{Competing interests}

The authors declare that they have no conflicts of interest. The authors alone are responsible for the content and writing of the paper.

\section{References}

1. Watson J. Oxidants, antioxidants and the current incurability of metastatic cancers. Open Biol 2013; 3: 120144.

2. Printz C. Is cancer research too conservative? Leaders weigh in on barriers to the fight against cancer. Cancer 2013; 119: 1605-6.

3. Esserman LJ, Thompsom IM, Reid B. Overdiagnosis and Overtreatment in Cancer an Opportunity for Improvement. Jama 2013; [Epub ahead of print].

4. Lanzafame RJ. Photobiomodulation: an enlightened path emerges. Photomed Laser Surg 2013; 31: 299-300.

5. Valastyan S, Weinberg RA. Tumor metastasis: molecular insights and evolving paradigms. Cell 2011;147: 275-92.

6. Santana-Blank L, Rodríguez-Santana E, Santana-Rodríguez JA, Santana-Rodríguez KE, Reyes H. Laser photobiomodulation as a potential multi-target anticancer therapy-review. Journal of Solid Tumors 2013; 3: 50-62.

7. Del Giudice E, Guiliani L. Coherence in water and kT coherence in living matter. In: Non Thermal Effects and Mechanisms of Interaction Between Electromagnetic Fields and Living Matter. L. Giuliani, and M. Soffritti (eds.). Bologna, Italy: Ramazini Institute. European Journal of Oncology Library 2010 ; 7-23.

ISSN 2330-4049 
8. Basset CA. Fundamental and practical aspects of therapeutic uses of pulsed electromagnetic fields. $J$ Cell Biochem. 1993; 51: 387-93.

9. Lanzafame RJ. Philosophy, dogma, and possibilities. Photomed Laser Surg 2012; 30: 403-4.

10. Lanzafame RJ. Photobiomodulation and cancer and other musings. Photomed Laser Surg 2011; 29: 3-4.

11. Karu T. Mitochondrial mechanisms of photobiomodulation in context of new data about multiple roles of ATP. Photomed Laser Surg 2010; 28: 159-60.

12. Santana-Blank LA, Rodríguez-Santana E, Vargas F, et al. Phase I trial of an infrared pulsed laser device in patients with advanced neoplasias. Clin Cancer Res 2002; 8: 3082-91.

13. Santana-Blank LA, Castes M, Rojas ME, Vargas F, Scott-Algara D. Evaluation of serum levels of tumour necrosis factor-alpha (TNF-alpha) and soluble IL-2 receptor (sIL-2R) and CD4, CD8 and natural killer (NK) populations during infrared pulsed laser device (IPLD) treatment. Clin Exp Immunol 1992; 90: 43-8.

14. Coussens LM, Zitvogel L, Palucka AK. Neutralizing Tumor-Promoting Chronic Inflammation: A Magic Bullet? Science 2013; 339: 286-91.

15. Tanaka Y, Matsuo K, Yuzuriha S, Yan H, Nakayama J. Non-thermal cytocidal effect of infrared irradiation on cultured cancer cells using specialized device. Cancer Sci 2010; 101: 1396-402.

16. Santana-Blank LA, Reyes H, Rodríguez-Santana E, Santana-Rodríguez KE. Microdensitometry of T2-weighted magnetic resonance (MR) images from patients with advanced neoplasias in a phase I clinical trial of an infrared pulsed laser device (IPLD). Lasers Surg Med 2004; 34: 398-406.

17. Malyarenko DI, Ross BD, Chenevert TL. Analysis and correction of gradient nonlinearity bias in apparent diffusion coefficient measurements. Magn Reson Med 2013; [Epub ahead of print].

18. Malyarenko D, Galbán CJ, Londy FJ, et al. Multi-system repeatability and reproducibility of apparent diffusion coefficient measurement using an ice-water phantom. J Magn Reson Imaging 2013; 37: 1238-46.

19. Abrahamse H. Regenerative medicine, stem cells, and low-level laser therapy: future directives. Photomed Laser Surg 2012; 30: 681-2.

20. Costa FF. Epigenomics in cancer management. Cancer Manag Res 2010; 2:255-65.

21. Joyce KM, Downes CS, Hannigan BM. Radioadaptation in indian muntjac fibroblast cells induced by low intensity laser irradiation. Mutat. Res 1999; 435: 35-42.

22. Strohman R. Maneuvering in the complex path from genotype to phenotype. Science 2002; 296: 701-3.
23. González JA, Martín-Landrove M, Carbo JR, Chacón M. Bifurcations and chaos of DNA solitonic dynamics, in: International Centre for Theoretical Physics bAU5 (eds.). Trieste, Italy: International Atomic Energy Agency and United Nations Educational Scientific and Cultural Organization 1994; $1-29$.

24. Gautam AP, Fernandes DJ, Vidyasagar MS, Maiya AG, Nigudgi S. Effect of low-level laser therapy on patient reported measures of oral mucositis and quality of life in head and neck cancer patients receiving chemoradiotherapy--a randomized controlled trial. Support Care Cancer 2013; 21: 1421-8.

25. Santana-Blank LA, Rodríguez-Santana E, Santana-Rodríguez KE. Photo-infrared pulsed bio-modulation (PIPBM): a novel mechanism for the enhancement of physiologically reparative responses. Photomed Laser Surg 2005; 23: 416-424.

26. Santana-Blank L, Rodríguez-Santana E, Santana-Rodríguez KE. Theoretic, experimental, clinical bases of the water oscillator hypothesis in near-infrared photobiomodulation. Photomed Laser Surg.2010; 28: S41-52.

27. Santana-Blank L, Rodríguez-Santana E, Santana-Rodríguez KE. Photobiomodulation of aqueous interfaces as selective rechargeable bio-batteries in complex diseases: personal view. Photomed Laser Surg 2012; 30: 242-249.

28. Karu T. Mitochondrial mechanisms of photobiomodulation in context of new data about multiple roles of ATP. Photomed Laser Surg. 2010; 28: 59-60.

29. Myakishev-Rempel M, Stadler I, Brondon P. et al. A preliminary study of the safety of red light phototherapy of tissues harboring cancer. Photomed Laser Surg 2012; 30: 551-8.

30. Tata DB, Waynant RW. Laser therapy: A review of its mechanism of action and potential medical applications. Laser \& Photonics Review 2011; 5: 1-12.

31. Luis Santana-Blank, Elizabeth Rodríguez-Santana, Jesús A. Santana-Rodríguez, Karin E. Santana-Rodríguez, Solid tumors and photobiomodulation: A novel approach to induce physiologically reparative homeostasis/homeokinesis-review. Journal of Solid Tumors 2012; 2: 23-35.

32. Pollack GH. The Fourth Phase of Water: Beyond Solid, Liquid, and Vapor. 1st ed. Seattle, WA: Ebner and Sons Publishers; 2013.

33. Santana-Blank L, Rodríguez-Santana E, Santana-Rodríguez JA, Santana-Rodríguez KE. Photobiomodulation of Aqueous Interfaces: Finding Evidence to Support the Exclusion Zone in Experimental and Clinical Studies. Photomedicine \& Laser Surgery 2013; In Press. 